\title{
Auricular acupressure as a complementary therapy for psoriasis vulgaris: study protocol for a multicenter randomized controlled trial
}

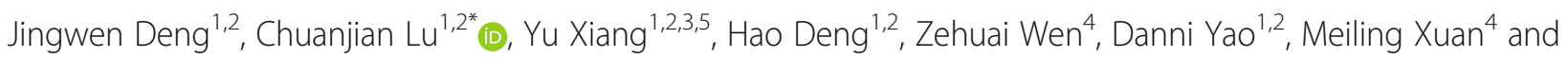
Yuhong Yan ${ }^{1,2}$

\begin{abstract}
Introduction: Psoriasis vulgaris is a common skin disease characterized by persistent localized erythematous scaly plaques, typically on the elbows, knees, and scalp. It is an immune-abnormal disease that progresses slowly over a long period with frequent symptom recurrence. Current studies have shown that acupuncture is an effective therapy for psoriasis. However, the scientific evidence of the efficacy of auricular acupressure treatment for patients with psoriasis is still insufficient. Therefore, we designed a randomized controlled clinical trial to investigate the effect, safety, and cost-effectiveness of auricular acupressure in addition to medication in patients with psoriasis.

Methods and analysis: This on-going study is a two-arm parallel, assessor-blinded, randomized controlled trial in which 180 participants with psoriasis will be recruited and then randomly allocated into two groups in a 1:1 ratio. Equal randomization will be conducted using a computer-generated random allocation sequence. Participants in the intervention group will receive auricular acupressure treatment once per week for 4 weeks, and calcipotriol betamethasone ointment for topical use once daily for 4 weeks. Participants in the control group will receive only calcipotriol betamethasone ointment treatment once daily for 4 weeks. All patients will be followed up for 12 weeks. The primary outcome is relapse rate. The secondary outcomes include time to relapse, rebound rate, time to new onset, Psoriasis Area and Severity Index score improvement rate, body surface area affected, a visual analogue scale, and Dermatology Life Quality Index. Cost-effectiveness analysis will be carried out from a health and community care provider perspective.
\end{abstract}

Discussion: This multicenter randomized controlled trial will provide important clinical evidence for the effect and safety of auricular acupressure as a complementary therapy in patients with psoriasis.

Trial registration: Chinese Clinical Trial Registry, ChiCTR-TRC-14004916. Registered on 20 May 2014. This protocol is version 3.0 which was updated on 24 September 2016.

\section{Background}

Psoriasis vulgaris is an immune-abnormal, chronic skin disease characterized by well-delineated red, scaly plaques. It is induced by a number of environmental factors and arouses great public concern because of its high prevalence,

\footnotetext{
* Correspondence: luchuanjian888@vip.sina.com

'Psoriasis Clinical and Basic Research Team, Guangdong Provincial Hospital of Chinese Medicine, Guangzhou 510120, China

${ }^{2}$ Guangdong Provincial Academy of Chinese Medical Sciences, Guangzhou 510120, China

Full list of author information is available at the end of the article
}

impact on life quality, and incurable characteristics [1]. With a prevalence of $1 \%$ to $3 \%$, psoriasis is likely to be encountered by general practitioners [2]. Furthermore, psoriasis has also been associated with a significantly increased risk of myocardial infarction, stroke, and peripheral vascular disease, possibly because of accelerated atherosclerosis in the setting of an inflammatory state [3].

Traditional Chinese medicine (TCM) is based on the fundamental principle of Yin-Yang balance, Five Elements, and a relationship between humans and nature

(c) The Author(s). 2019 Open Access This article is distributed under the terms of the Creative Commons Attribution 4.0 International License (http://creativecommons.org/licenses/by/4.0/), which permits unrestricted use, distribution, and reproduction in any medium, provided you give appropriate credit to the original author(s) and the source, provide a link to the Creative Commons license, and indicate if changes were made. The Creative Commons Public Domain Dedication waiver (http://creativecommons.org/publicdomain/zero/1.0/) applies to the data made available in this article, unless otherwise stated. 
[4]. When treating diseases, TCM first evaluates presenting symptoms to differentiate the syndrome related to the disease and then clarifies a therapeutic method for this disease. For more than 2000 years, TCM has been used to treat various diseases in China and throughout East Asia, and it still remains the first choice of treatment for many people because its effectiveness and its cost being inexpensive. Clinical practices have also proved that TCM is beneficial and effective in alleviating clinical symptoms, improving quality of life, immune function, reducing metastasis, and preventing recurrence in various diseases [5-10].

In the perspective of TCM, the pathogenesis of psoriasis is deficiency in origin and excess in symptom (Ben Xu Biao Shi), and in most cases it co-occurs with blood stasis. In chronic psoriasis, $q i$ was exhausted during a long disease course and then the blood circulation was disturbed. Finally, $q i$ and blood coagulated and blocked the meridians, which led to lack of nourishment for the skin and muscle. Thus, TCM clinical experts advocated 'treatment of psoriasis from the blood aspect' [11, 12]. The effective ways to achieve this are blood-activating herbal medicines and treatments such as acupuncture and bloodletting. A systematic review indicated that the evidence for the effect of multi-herb formulations for psoriasis was promising in a number of the studies [13]. A meta-analysis reported that Chinese medicine was effective for psoriasis vulgaris since it is noninferior to acitretin and it could produce add-on effects when combined with acitretin, as well as reducing the acitretin-induced adverse events [14]. Chinese medicine combined with narrowband ultraviolet B for treating psoriasis also showed improved efficacy $[15,16]$. Herbal formulae or plant extract topical management were analyzed in other systematic reviews, which showed that topical herbal formulae or plant extracts could improve overall clinical symptoms [17-19].

Acupuncture is a typical representative of TCM, and is one of the various general therapies for psoriasis. In a systematic review, acupuncture therapies show some evidence of benefit for the treatment of psoriasis [20]. Auricular acupressure is the acupuncture-related practice. It is a noninvasive form of acupuncture which uses physical pressure applied to the auricular acupressure point (AAP) by $\mathrm{Vac}$ caria seeds [21]. The prescription component of auricular acupressure is principally based on the divisions of the auricle, with definite terms and denoting s [22]. Named AAPs and regions are distributed over the entire surface of the auricle (Fig. 1).

Auricular acupressure is characterized by balancing Yin and Yang, dredging meridians and collaterals, regulating the functions of organs, and building up body resistance to pathogenic factors [23]. Auricular acupressure has been used to treat various diseases. In recent studies, auricular acupressure has been shown to help menstruating women effectively relieve menstrual headache [24]. Furthermore,

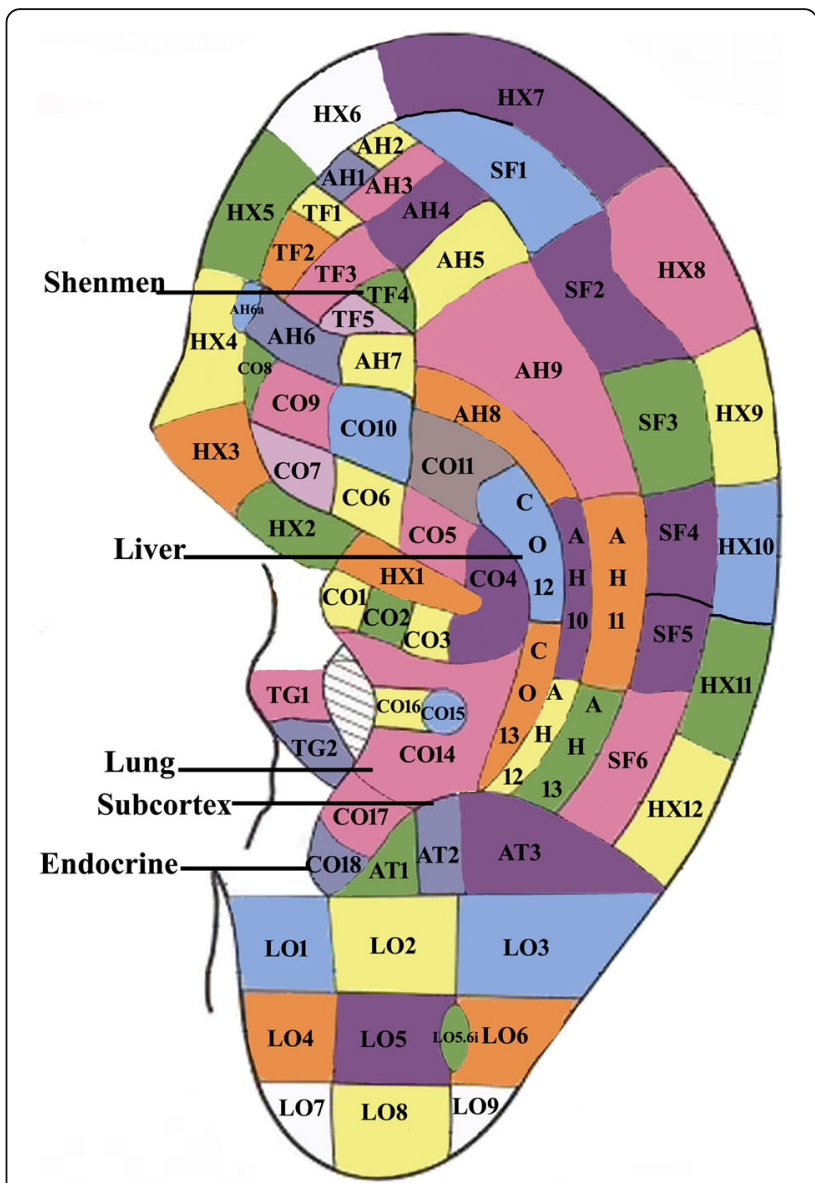

Fig. 1 Standard codes for the division of the auricle

it has been shown that auricular acupressure treatment can improve sleep quality and daytime dysfunction in insomnia patients [25-27]. By stimulating a point on the ear, a promising therapeutic effect is believed to occur on the gross anatomical organ associated with that point. Studies have demonstrated that auricular therapy can promote general $q i$ and blood circulation; hence, it can eventually improve the blood flow [28].

Since there is little experience using auricular acupressure in psoriasis, we aim to analyze the additional efficacy on symptom improvement and relapse rate associated with auricular acupressure treatment for psoriasis. The aim of our study is to show whether there is additional benefit in improving symptoms and preventing recurrence when adding auricular acupressure treatments to usual therapy compared with usual therapy alone for people with psoriasis.

\section{Methods/design}

Study design

This study is a two-parallel arm, assessor-blinded, randomized controlled trial. The trial will be conducted by Guangdong Provincial Hospital of Chinese Medicine 
(GPHCM) and performed at two clinical research centers in China (Guangdong Provincial Hospital of Chinese Medicine and Guangzhou Red Cross Hospital) in accordance with the Declaration of Helsinki and the Guidelines for Good Clinical Practice.

This study will select patients with blood stasis syndrome on a TCM diagnosis based on a TCM syndrome differentiation standard [29,30]. Eligible participants will be stratified by age and sex and randomly allocated in a 1:1 ratio to one of the treatment arms (the auricular acupressure plus calcipotriol betamethasone ointment group or the control calcipotriol betamethasone ointment group) and receive treatment for 4 weeks, with 12 weeks of follow-up (Fig. 2). The Standard Protocol Items: Recommendations for Interventional Trials (SPIRIT) checklist is provided in Additional file 1.

\section{Participants}

\section{Inclusion criteria}

A total of 180 patients will be recruited from the outpatient clinics of the two hospitals. The eligibility criteria for the study include: 1) those with a diagnosis of psoriasis vulgaris (referring to the 2008 clinical guidelines of psoriasis reported by the Chinese Medical Association [31]; 2) aged 18 to 70 years; 3 ) psoriasis vulgaris patients (Psoriasis
Area and Severity Index score (PASI) score of 7-20, with body surface area (BSA) affected $<30 \%$ ); and 4 ) willing to sign a written informed consent.

\section{Exclusion criteria}

Participants will be excluded if they are experiencing or have a history of the following: 1) psoriatic arthritis, guttate psoriasis, inverse psoriasis or exclusively involving the face; 2) pregnancy, breast-feeding, or those who intend to become pregnant within 1 year; 3) a Self-rating Anxiety Scale (SAS) $>50$ or Selfrating Depression Scale (SDS) $>53$, or with other psychiatric disorders; 4) important systemic disease that cannot be controlled through common treatment (either with infection, electrolyte imbalance, acid-base disturbance, calcium metabolic disorder, or cancer; 5) those allergic to any medicine or ingredients used in this study; 6) those participating in other clinical trials or those who have participated within 1 month before entry into the study; 7) those who have been treated with topical treatments (i.e., corticosteroids, retinoic acid) within 2 weeks, or systemic therapy or phototherapy (ultraviolet B) and psoralen combined with ultraviolet A within 4 weeks, or biological therapy within 12 weeks before beginning the study.

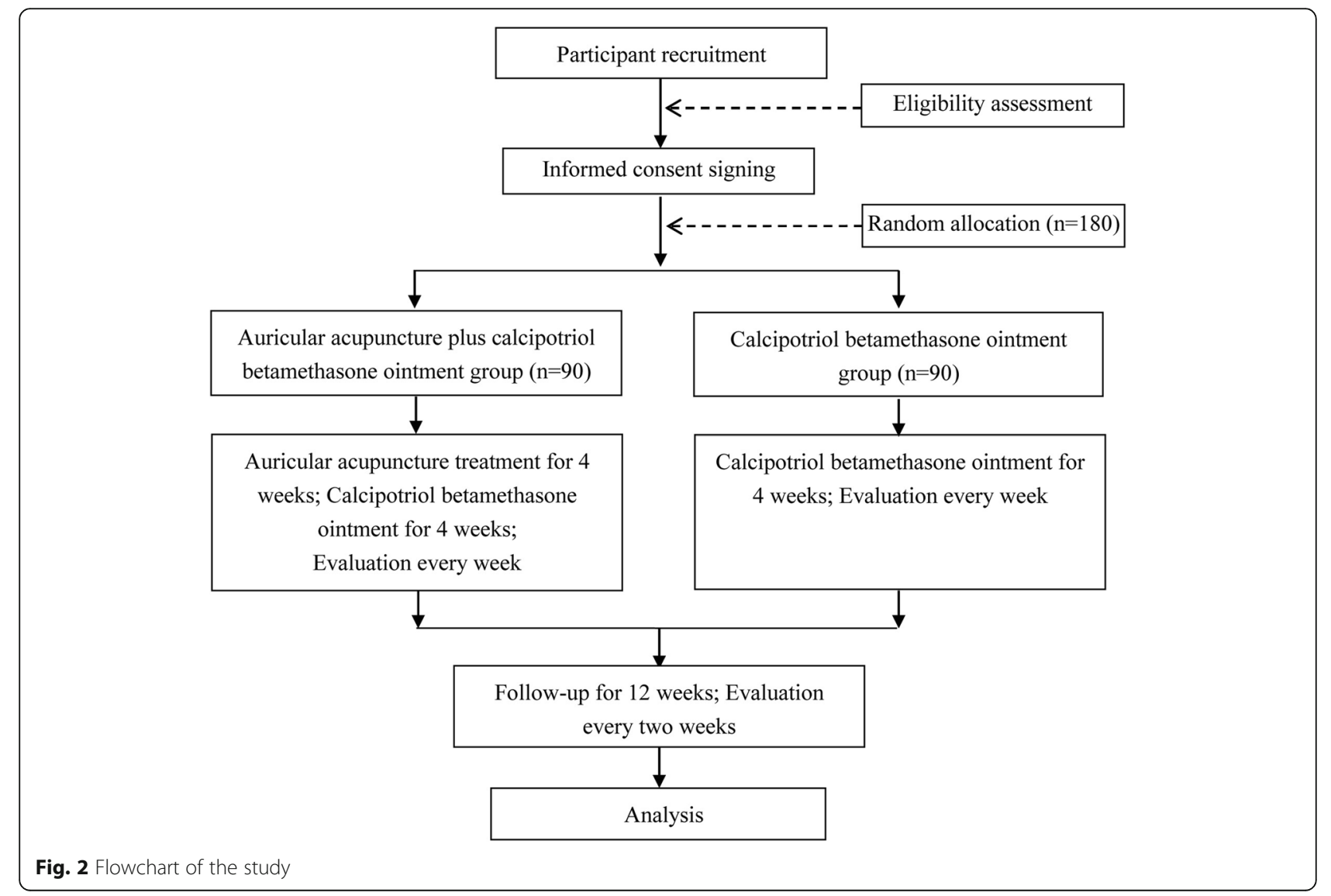




\section{Randomization}

Eligible patients will be randomly assigned in a 1:1 ratio to one of the two groups (the treatment group who receive auricular acupressure plus calcipotriol betamethasone ointment or the control group who receive calcipotriol betamethasone ointment) at the second visit through central randomization. Equal randomization will be conducted using a computer-generated random allocation sequence through the stratified block randomization method of the SAS software (version 9.12; SAS Institute, Inc., Cary, NC, USA) with a block size of 4 by the Key Unit of Methodology in Clinical Research (KUMCR) of Guangdong Provincial Hospital of Chinese Medicine. Allocation concealment will be ensured since the randomization code will be released by the Interactive Web Response System for Chinese Medicine Trials (IWRS-CMT), which is a verified online randomization facility established by the KUMCR (http:// www.gztcmgcp.net/sjxt/login.asp). Following this, the participants will be randomly allocated to the two different groups. The practitioners will be aware of the allocation arm according to the different medical procedures. However, the evaluation of participants and the analysis of the results will be performed by physician assessors and statisticians who are blinded to the group allocation [32].

\section{Intervention}

Participants in both groups will be provided with calcipotriol betamethasone ointment. Subjects randomized to the experimental group will receive integrated auricular acupressure therapy and calcipotriol betamethasone ointment topical therapy.

\section{Auricular acupressure treatment}

Patients in the auricular acupressure plus calcipotriol betamethasone ointment group will receive auricular acupressure treatment once a week for 4 weeks. At every visit, therapists treat the patients with auricular acupressure. All of the therapists participated in this trial will be standardized in their training, including in the study protocol and methods of treatment. We selected auricular acupoints as suggested in the nomenclature and location of auricular points [33]. The auricular acupoints are based on a set of anatomical maps superimposed onto the ear. The following auricular points will be used for treatment: Lung (Fei, CO14), Shenmen (TF4), endocrine (CO18), Subcortex (AT4) and Liver (Gan, CO12) (Fig. 1). According to modern research and Chinese medicine, "The liver (Gan) stores blood. Liver dispersion, qi stagnation', 'The lung (Fei) governs skin and hair,' and, in addition to treating respiratory-related disease, the auricular points Liver (CO12) and Shenmen (TF4) work on regulating emotion and reducing stress, anxiety, and excessive sensitivity [34, 35]. Lung (CO14) also works on relieving painful and itchy skin diseases [36]. Endocrine (CO18) and Pizhixia
(AT4) could be helpful with endocrine hormone balance, hypersensitivity, and rheumatism [37, 38].

After sterilization with $75 \%$ alcohol, Vaccaria seeds will be stuck and fixed on the above auricular points of both ears, with each pressed individually for $1 \mathrm{~min}$ to induce stimulus until patients feel endurable heat and distending pain on the auricles, and patients are advised to repeat the pressing themselves four times daily for 5 consecutive days and then to remove the seeds stuck to their auricles on the morning of day 6 , which is helpful to avoid contact dermatitis caused by long-term adhesive tape.

\section{Calcipotriol betamethasone ointment treatment}

Traditional topical therapies (such as corticosteroids, vitamin D and analogues, dithranol, and tar preparations) are recommend by NICE guideline as first-line therapy [39]. In this study, we used a routine drug for first-line therapy in the control group and as a base treatment in the intervention group. Thus, participants in both groups will be provided with calcipotriol betamethasone ointment (Daivobet $^{\circ}$ gel; calcipotriol $50 \mu \mathrm{g} / \mathrm{g}$ plus betamethasone 0.5 $\mathrm{mg} / \mathrm{g}$ ) for topical use once daily until the PASI score of the patient is reduced to 0 . However, the course of calcipotriol betamethasone ointment treatment would last no more than 4 weeks.

\section{Outcome}

\section{Primary outcome measurement}

The primary outcome measure in the trial is relapse rate in the treatment period and follow-up period. Relapse is defined as losing $50 \%$ of the improvement obtained from treatment once the treatment is stopped [40]. The PASI will be assessed every visit during the treatment period and the follow-up period. Meanwhile, patients will be required to report the emergence of a variety of conditions at any time in the study period. Assessors will evaluate the PASI score on the same or closest day. Target lesions will be recorded as digital photographs by single-lens reflex (SLR) cameras at every visit.

\section{Secondary outcome measurements}

Secondary outcome measures include the time to relapse, rebound rate, time to new onset, PASI improvement rate, BSA affected, a visual analog scale (VAS), and Dermatology Life Quality Index (DLQI). Time to relapse is defined as the time it takes to lose $50 \%$ of the improvement obtained from treatment (Table 1). Time to new onset is the PASI score improvement achieved (PASI-50) for the first time, but a failure to keep the PASI-50 improvement all through the treatment period. Rebound is defined as a PASI score of $125 \%$ of that at baseline, or the occurrence of new generalized pustular, erythrodermic, or more inflammatory psoriasis occurring [41]. The VAS and BSA will be assessed every week during the first 4 weeks and 
every 2 weeks in the follow-up period. The DLQI will be self-assessed by patients every 4 weeks throughout the trial. Laboratory reports will be also monitored until the last visit. TCM syndrome is one of the assessments which will be recorded before and after the auricular acupressure, as well as at the end of the follow-up period (Table 2)

\section{Health economics}

Economic evaluation will be carried out from the perspective of the Health Department of Guangdong Province, which will be in the form of a cost-utility analysis conducted using utility values obtained from the DLQI preferencebased quality of life measure. DLQI is a dermatologyspecific quality of life instrument for routine clinical use. It is a validated questionnaire with 10 simple questions. At present, the DLQI is the most frequently used instrument for evaluating the impact of skin disease and related treatment on the lives of patients. The DLQI will be measured at baseline and at 4 and 16 weeks for utility-based quality of life evaluation in this study. Resource use will include intervention costs, healthcare costs, and community service costs, which will be calculated for each trial participant. We will analyze an incremental cost-effectiveness ratio (ICER) of cost per patient by calculating the incremental mean difference in costs between the two trial arms and incremental difference in patient outcome after the follow-up.

\section{Sample size}

There is a growing body of research evaluating the effect of auricular acupressure for various diseases. However, there is still a lack of studies evaluating the effect of auricular acupressure on psoriasis. There is no previous study on which to base the sample size calculation. Thus, we used the primary outcome relapse rate to determine a sample size calculation. Based on the study of Menter et al. [42], the relapse rate of calcipotriol betamethasone ointment topical therapy after 4 weeks of treatment and 4 weeks of follow-up is $82 \%$. We assume that the relapse rate of auricular acupressure therapy combined with calcipotriol betamethasone ointment topical therapy for psoriasis in week 12 is $20 \%$ less than topical therapy alone and that the relapse rate of calcipotriol betamethasone ointment topical therapy is $82 \%$. On the basis of this hypothesis, and in order to compare the two groups for a significance level $\alpha=0.05$ and a power $1-\beta=0.80$, we calculated the sample size by PASS statistics software (version 11.0.10; NCSS LLC., Utah, USA); 77 patients with psoriasis would have been required in each group to achieve $80 \%$ power to detect a difference between the group proportions of -0.2 . The test statistic used is the two-sided $Z$ test with pooled variance. The significance level of the test is targeted at 0.05 . Considering 15\% loss to follow-up, 90 patients are needed in each arm, totaling 180 patients in all.

\section{Data collection}

Outcome measurements will be carried out and recorded using paper case report forms (CRF) and checked for each participant at every visit by a certificated clinical researcher. To promote patient enrolment, retention, and completion of follow-up, all the treatments and laboratory

Table 1 Summary of measurement

\begin{tabular}{|c|c|c|}
\hline Outcome & Measurement tool & Details \\
\hline \multicolumn{3}{|l|}{ Primary outcome } \\
\hline Relapse rate & $\begin{array}{l}\text { Psoriasis Area and Severity Index } \\
\text { (PASI) score }\end{array}$ & $\begin{array}{l}\text { Relapse defined as losing } 50 \% \text { of the improvement obtained from treatment once the } \\
\text { treatment is stopped }\end{array}$ \\
\hline \multicolumn{3}{|l|}{ Secondary outcome } \\
\hline Time to relapse & PASI score & $\begin{array}{l}\text { Time domain between achieving at least } 50 \% \text { reduction in PASI score and losing } 50 \% \text { of the } \\
\text { improvement obtained from treatment once the treatment is stopped. }\end{array}$ \\
\hline $\begin{array}{l}\text { Time to new } \\
\text { onset }\end{array}$ & PASI score & Time domain between lesion clearing and recurrence \\
\hline Rebound rate & PASI score & $\begin{array}{l}\text { Rebound defined as a severe and sudden change in the severity of psoriasis that is } \\
\text { significantly worse than before the treatment was initiated }\end{array}$ \\
\hline $\begin{array}{l}\text { PASI } \\
\text { improvement } \\
\text { rate }\end{array}$ & PASI score & $\begin{array}{l}\text { PASI score is a tool used to measure the severity and extent of psoriasis. It takes a few } \\
\text { minutes and experience to calculate it accurately. A representative area of psoriasis is } \\
\text { selected for each body region. The intensity of redness, thickness, and scaling of the } \\
\text { psoriasis is assessed as none (0), mild (1), moderate (2), severe (3), or very severe (4) }\end{array}$ \\
\hline VAS & Visual analog scale (VAS) & $\begin{array}{l}\text { VAS is the most common pain scale for quantification of endometriosis-related pain. We use } \\
\text { VAS to evaluate the feeling of pruritus in participants during the treatment period }\end{array}$ \\
\hline BSA & Body surface area (BSA) & $\begin{array}{l}\text { BSA is a common measure in the medical field and part of the complete body size and } \\
\text { composition profile }\end{array}$ \\
\hline DLQI & $\begin{array}{l}\text { Dermatology Life Quality Index } \\
\text { (DLQI) }\end{array}$ & $\begin{array}{l}\text { DLQI is a dermatology-specific quality of life instrument. It is a simple 10-question validated } \\
\text { questionnaire. At present, the DLQI is the most frequently used instrument in studies of } \\
\text { randomized controlled trials in dermatology }\end{array}$ \\
\hline
\end{tabular}


Table 2 Schedule for treatment and outcome measurements

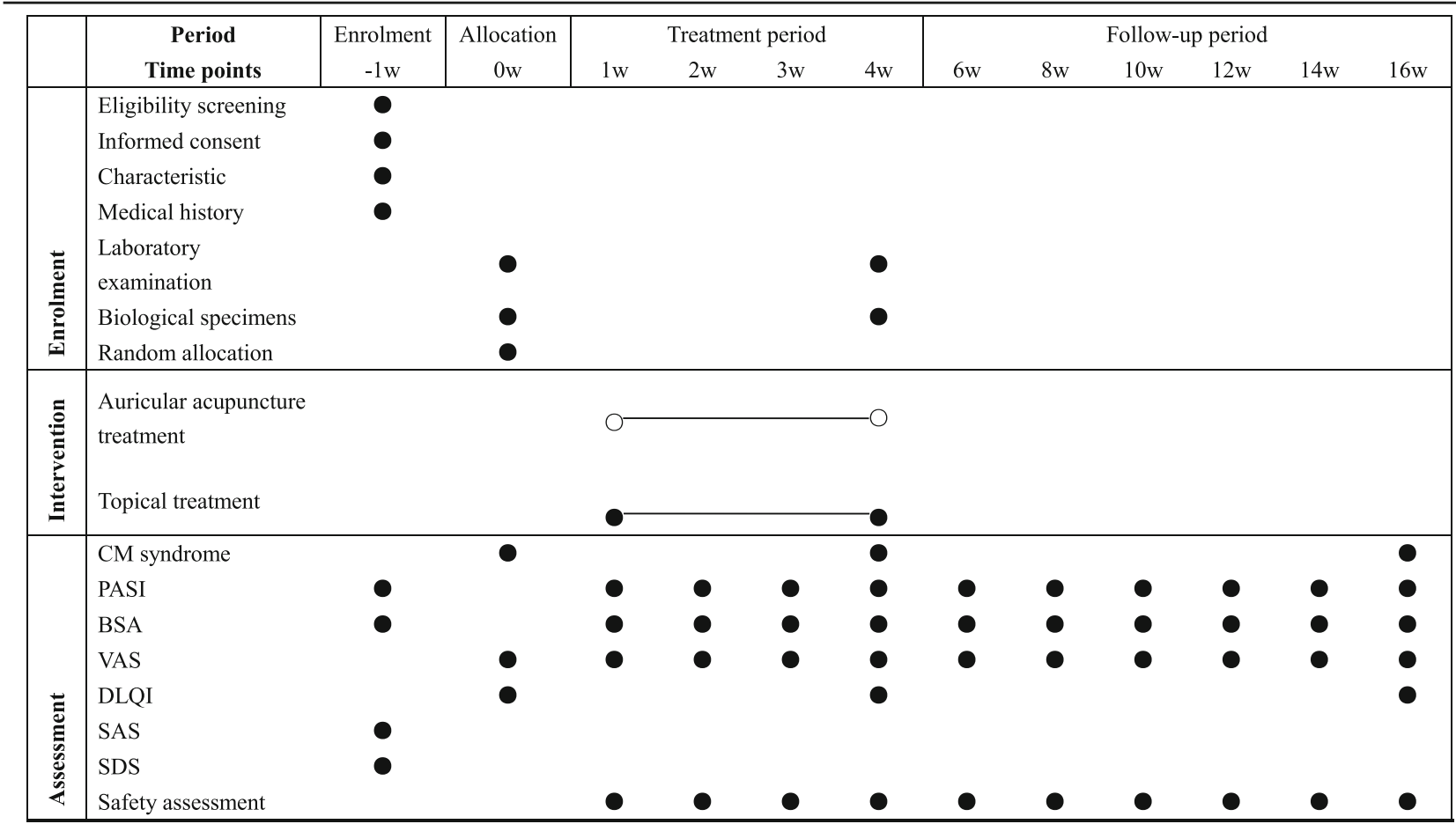

: Both the auricular acupuncture group and the usual care group.

$\bigcirc$ : Only the auricular acupuncture group.

BSA body surface area, CM Chinese medicine, DLQI Dermatology Life Quality Index, PASI Psoriasis Area and Severity Index, SAS Self-rating Anxiety Scale, SDS Selfrating Depression Scale, VAS visual analog scale

tests will be free. A 300 RMB gift will be sent to participants when they have completed the follow-up.

\section{Data management}

Data managers will enter the information on the CRF into an electronic database using the double-entry method. There is no exclusive trial steering committee named in this trial. The integrity of CRF data in different centers will be monitored regularly by Guangdong International Clinical Research Center of Chinese Medicine (Guangzhou, China). As well as the safety data, and the critical efficacy outcomes will be assessed by the Data Monitoring Committee from GPHCM.

Patients will be pseudonymized by study identification numbers for participant confidentiality. Access to the CRF database will be limited to the study group and the coordinator.

\section{Statistical analysis}

All analyses will be performed with PASS Statistics and SAS 9.2 software by a statistician who is blinded to the random allocation of groups. An intent-to-treat (ITT) basis statistical analysis with a 95\% confidence interval will be performed using multiple imputations. The major imputation is multiple imputation by chained equations (MICE). The ITT analysis will include all patients who are randomized [43]. Safety analysis will be undertaken by analyzing the frequency of adverse events which are suspected as related to the treatment. Planned subgroup analyses include those based on the type of TCM syndromes and severity of psoriasis. The various parameters observed will be compared using a Chi-square test for noncontinuous variables (i.e., the primary outcome relapse rate) and $t$ test and analysis of variance (ANOVA) for continuous variables. In order to distinguish the treatment effect and time effect, the repeated measures ANOVA change from baseline will be performed for the different time point assessments. Statistical significance is established at $P<0.05$. No formal interim analysis is planned.

\section{Adverse events}

Before the start of treatment and after 4 weeks of treatment a medical history will be recorded for each patient, and standard laboratory examinations and specific laboratory investigations will also be performed. The standard laboratory examinations include: hematologic parameter assessments (hemoglobin, red blood cells, platelets, and white blood cell counts); urinalysis (proteins, red blood cell and white blood cell biochemical assessment (serum electrolytes)); indices of renal function (creatinine, urea) and hepatic function (alkaline phosphatase, aspartate aminotransferase, alanine aminotransferase, and gamma-glutamyl transpeptidase); serum 
calcium; and an electrocardiogram. The specific laboratory investigations mainly include serum cytokine levels.

All adverse events will be collected and graded for severity and potential relation to the treatments in the study by assessors at every visit. Safety evaluations include the incidence of treatment-induced or serious adverse events, dropout because of adverse events, and changes from baseline of the PASI score and laboratory parameters. Patients will be also asked about other adverse effects of auricular therapy such as faintness, nausea, and vomiting. In case of severe adverse effects, auricular therapy will be discontinued immediately.

\section{Discussion}

Psoriasis is a chronic inflammatory disease that manifests as a wide spectrum of clinical signs ranging from variable skin symptoms to arthritis. Effective treatments are available for the routine care of individuals with psoriasis. Various therapeutic regimens including different topical corticosteroids, topical vitamin D analogs, biological agents, phototherapy, photochemotherapy, cyclosporin, systemic therapy with methotrexate, and combination therapies have shown beneficial therapeutic effects for patients with psoriasis [44]. However, some of these treatments are expensive, some require appropriate monitoring, and some may only be accessed in clinical care settings because of potential adverse events. However, with there being no cure, the aim of therapies for people with psoriasis is to minimize the extent and severity of the disease so that it no longer substantially impacts their quality of life. Evidence indicates that, currently, a substantial proportion of patients are dissatisfied with their treatment for psoriasis. Besides, patients who discontinue treatments may experience a return of the disease or worsening of the disease [45]. This detrimental impact on quality of life is a relentless condition yet one for which many people have given up seeking any medical support.

Hence, relapses are common in psoriasis and patients may have to maintain therapy for long durations. However, the pattern of relapse varies. Some patients have early and frequent relapses and others may have long-term remissions with infrequent relapses [46]. In the study by Kaur et al. the duration of remissions varied widely from 2 weeks to 9 years, and $4 \%$ of patients never had complete remission in India [47]. Studies on cyclosporine in psoriasis found that $50-60 \%$ of patients relapsed 6 months after treatment withdrawal and that the time to relapse depended on the severity of disease, the dose required to achieve clearance, and the extent of clearing achieved before termination of the drug [48]. In the study by Heydendael et al. PASI-75 was achieved in $60 \%$ of patients with cyclosporine treatment, but discontinuation often led to a relapse [49].
Consequently, to find more effective therapeutic methods to prevent the return of disease activity many individuals have turned their attention to treatments such as TCM. Currently, clinical experience has been accumulated for TCM treatment of psoriasis [13-19, 50-52]. Most significantly, as a type of TCM, acupuncture has shown respectable efficacy and is broadly accepted internationally.

Auricular acupressure is defined as a healthcare modality whereby the external surface of the ear, or auricle, is stimulated to alleviate pathological conditions in other parts of the body [52]. The auricular acupoints are based on a set of anatomical maps superimposed onto the ear. The stimulation of auricular acupoints is intended to regulate $q i$, activate the meridians, and is proposed to affect the gross anatomical organ associated with that point. In so doing, a variety of health problems (for example, chronic pain, insomnia, and lactation disorder) have been successfully cured [53-56]. Systematic reviews of acupuncture therapy for psoriasis indicated that acupuncture-related techniques could be considered as an alternative or adjuvant therapy for psoriasis $[20,57,58]$. We found some evidence of a benefit of auricular acupressure for the treatment of psoriasis vulgaris [59, 60]. However, the conclusions are limited by the small number of included trials from single studies. We aim to clarify the effect of auricular acupressure for psoriasis but are unable to find any previous published studies that addressed this question. Thus, the present study is designed to look at the relapse rate in psoriasis with auricular acupressure plus usual treatment after achieving PASI-50, and it may contribute to this aim when auricular acupressure is used in addition to medication in the present design.

The primary aim of the present study is to evaluate the control of psoriasis recurrence after auricular acupressure plus usual treatment in patients. The secondary aim is to evaluate the effect of the auricular acupressure plus usual treatment versus usual treatment alone on $\mathrm{cu}$ taneous symptom reduction at 4 weeks. We hypothesize that the combined treatment group will contain a higher proportion of patients who are better maintaining their recover status after achieving PASI-50, and who have a greater reduction in cutaneous symptoms at 4 weeks, compared with the usual treatment group. We are unable to find any previously published studies that addressed these questions. This study is one of the first trials to evaluate the effect of auricular acupressure on psoriasis. It may contribute to the aims described above when auricular acupressure is used in addition to medication.

\section{Trial status}

The recruitment phase began in March 2013. Thus far, 154 patients have been recruited. 


\section{Additional file}

Additional file 1: SPIRIT 2013 checklist: recommended items to address in a clinical trial protocol and related documents. (DOC $126 \mathrm{~kb}$ )

\section{Abbreviations \\ AAP: Auricular acupressure point; ANOVA: Analysis of variance; BSA: Body surface area; CRF: Case report forms; DLQI: Dermatology Life Quality Index; GPHCM: Guangdong Provincial Hospital of Chinese Medicine; ICER: Incremental cost-effectiveness ratio; ITT: Intent-to-treat; IWRS- CMT: Interactive Web Response System for Chinese Medicine Trials; KUMCR: Key Unit of Methodology in Clinical Research; MICE: Multiple imputation by chained equations; PASI: Psoriasis Area and Severity Index; SAS: Self-rating Anxiety Scale; SDS: Self-rating Depression Scale; \\ TCM: Traditional Chinese medicine; VAS: Visual analog scale}

\section{Authors' contributions}

JD drafted the manuscript. CL, ZW, and MX participated in the design of the study, YX, JD, DY, and YY coordinated the study. All authors read and approved the final manuscript.

\section{Funding}

This research was supported by a grant from the National Key Technology R\&D Program for the 12th Five-year Plan of Ministry of Science and Technology, China (no. 2013BAI02B03) and the Traditional Chinese Medicine Bureau Foundation of Guangdong Province (no. 20161111 and no. 20183005).

\section{Availability of data and materials} Not applicable.

\section{Ethics approval and consent to participate}

This research protocol had been reviewed and approved by the institutional review boards of both trial centers (GPHCM and Guangzhou Red Cross Hospital; B2012-55-01). Important protocol modifications will be immediately communicated to the GPHCM Ethics Committee and Data Monitoring Committee by amendments. All modifications will also be updated on the Chinese Clinical Trial Registry.

The data and findings will be disseminated to the public through patient associations, conference presentations, and open-access journals. The aggregated data will also be shared with the public through the Chinese Clinical Trial Registry.

Written informed consent will be obtained from all eligible patients before the enrollment and then taken by the treating clinician.

\section{Consent for publication}

Not applicable.

\section{Competing interests}

The authors declare that they have no competing interests.

\section{Author details}

${ }^{1}$ Psoriasis Clinical and Basic Research Team, Guangdong Provincial Hospital of Chinese Medicine, Guangzhou 510120, China. ${ }^{2}$ Guangdong Provincial Academy of Chinese Medical Sciences, Guangzhou 510120, China. ${ }^{3}$ Guangdong Provincial Key Laboratory of Clinical Research on Traditional Chinese Medicine Syndrome, Guangzhou 510120, China. ${ }^{4}$ Key Unit of Methodology in Clinical Research, Guangdong Provincial Hospital of Chinese Medicine, Guangzhou 510120, China. ${ }^{5}$ School of Medical Information Engineering, Guangdong Pharmaceutical University, Guangzhou 510006, China.

Received: 14 November 2017 Accepted: 27 May 2019

\section{Published online: 17 June 2019}

\section{References}

1. Lu CJ, Yu JJ, Deng JW. Disease-syndrome combination clinical study of psoriasis: present status, advantages, and prospects. Chin J Integr Med. 2012;18(3):166-71
2. Velez NF, Wei-Passanese EX, Husni ME, Mody EA, Qureshi AA. Management of psoriasis and psoriatic arthritis in a combined dermatology and rheumatology clinic. Arch Dermatol Res. 2012;304(1):7-13.

3. Menter A, Gottlieb A, Feldman SR, Van Voorhees AS, Leonardi CL, Gordon KB, et al. Guidelines of care for the management of psoriasis and psoriatic arthritis: section 1. Overview of psoriasis and guidelines of care for the treatment of psoriasis with biologics. J Am Acad Dermatol. 2008;58(5):826-50.

4. Xiwen L. Huangdi Neijing. China Press of Traditional Chinese Medicine; 2009. p. p68.

5. Xiaoging F, Fanli M, Dahui W, et al. Perceptions of traditional Chinese medicine for chronic disease care and prevention: a cross-sectional study of Chinese hospital-based health care professionals. BMC Complement Altern Med. 2018;18(1):209

6. Chao J, Dai Y, Verpoorte R, et al. Major achievements of evidence-based traditional Chinese medicine in treating major diseases. Biochem Pharmacol. 2017:139:94-104.

7. Cai Y, Boyd DL, Coeytaux RR, et al. Treatment of chronic conditions with traditional Chinese medicine: findings from traditional Chinese medicine hospitals in Hubei, China. J Altern Complement Med. 2015;21(1):40-5.

8. Wang $X$, Pi Y, Chen $B$, et al. Effect of traditional Chinese exercise on the quality of life and depression for chronic diseases: a meta-analysis of randomised trials. Sci Rep. 2015;5:15913.

9. Xing $Y$, Xiong $X$, Shang $H$, et al. The role of biologically active ingredients from Chinese herbal medicines in the regulation of autophagy in treating cardiovascular diseases and other chronic diseases. Curr Pharm Des. 2017; 23(7):1060-9.

10. Ming $H$, Sha $L, H o r ~ T$, et al. Current status of herbal medicines in chronic liver disease therapy: the biological effects, molecular targets and future prospects. Int J Mol Sci. 2015:16(12):28705-45.

11. Xue CC, Lu C. Evidence-based clinical Chinese medicine - volume 2: psoriasis vulgaris. Singapore: World Scientific Publishing; 2016. p. p26-9.

12. Meng S, Lin Z, Wang Y, et al. Psoriasis therapy by Chinese medicine and modern agents. Chin Med. 2018;13(1):16.

13. May BH, Zhang AL, Zhou W, et al. Oral herbal medicines for psoriasis: a review of clinical studies. Chin J Integr Med. 2012;18:172-8.

14. Zhang CS, Yang L, Zhang AL, et al. Is oral Chinese herbal medicine beneficial for psoriasis vulgaris? A meta-analysis of comparisons with acitretin. J Altern Complement Med. 2016;22:174-88.

15. Yang L, Zhang CS, May B, et al. Efficacy of combining oral Chinese herbal medicine and NB-UVB in treating psoriasis vulgaris: a systematic review and meta-analysis. Chin Med. 2015;10:27.

16. Yu JJ, Zhang CS, Zhang AL, et al. Add-on effect of Chinese herbal medicine bath to phototherapy for psoriasis vulgaris: a systematic review. Evid Based Complement Alternat Med. 2013;2013:673078.

17. Deng S, May BH, Zhang AL, et al. Topical herbal formulae in the management of psoriasis: systematic review with meta-analysis of clinical studies and investigation of the pharmacological actions of the main herbs. Phytother Res. 2014;28:480-97.

18. Deng S, May BH, Zhang AL, et al. Plant extracts for the topical management of psoriasis: a systematic review and meta-analysis. $\mathrm{Br} J$ Dermatol. 2013;169:769-82.

19. Deng S, May BH, Zhang AL, et al. Topical herbal medicine combined with pharmacotherapy for psoriasis: a systematic review and meta-analysis. Arch Dermatol Res. 2013:305:179-89.

20. Coyle M, Deng J, Zhang AL, Yu J, Guo X, Xue CC, Lu C. Acupuncture therapies for psoriasis vulgaris: a systematic review of randomized controlled trials. Forsch Komplementmed. 2015;22(2):102-9.

21. Lee EJ, Frazier SK. The efficacy of acupressure for symptom management: a systematic review. J Pain Symptom Manag. 2011;42(4):589-603.

22. World Federation of Acupuncture-Moxibustion Societies (WFAS). Auricular acupuncture point (WFAS STANDARD-002: 2012). World J Acupunct Moxibustion (WJAM). 2013;23:3 30th.

23. Huang L. Auricular acupoint therapeutics. Scientific and Technical Documentation Press; 2017. p. p17.

24. Sun LH, Li XH, Li WL, Liu L, Ma HL, Liang YL. Body acupuncture combined with auricular acupressure for menstrual headache: a randomized controlled clinical trial (Chinese). Zhen Ci Yan Jiu. 2015:40(1):70-4.

25. King HC, Spence DL, Hickey AH, Sargent P, Elesh R, Connelly CD. Auricular acupuncture for sleep disturbance in veterans with post-traumatic stress disorder: a feasibility study. Mil Med. 2015;180(5):582-90. 
26. Lan Y, Wu X, Tan HJ, Wu N, Xing JJ, Wu FS, et al. Auricular acupuncture with seed or pellet attachments for primary insomnia: a systematic review and meta-analysis. BMC Complement Altern Med. 2015;15(1):103.

27. Yang JL, Zhang R, Du L, Yang YS, Liu XC. Clinical observation on the neurotransmitters regulation in patients of insomnia differentiated as yang deficiency pattern treated with warm acupuncture and auricular point sticking therapy (Chinese). Zhongguo Zhen Jiu. 2014;34(12):1165-8.

28. Asher GN, Jonas DE, Coeytaux RR, Reilly AC, Loh YL, Motsinger-Reif AA, Winham SJ. Auriculotherapy for pain management: a systematic review and meta-analysis of randomized controlled trials. J Altern Complement Med. 2010;16:1097-108.

29. Guangdong Traditional Chinese Medicine Standardization Technical Committee. Psoriasis vulgar syndrome differentiation standard of traditional Chinese medicine (DB44/T 1423-2014). 2014

30. Xie X, Lui $C$, Zeng Z, et al. Research on the psoriasis vulgaris syndrome differentiation standard of traditional Chinese medicine based on data mining technology. In: 2013 IEEE International Conference on Bioinformatics and Biomedicine; 2013. p. 14079580

31. Psoriasis Group of Dermatology and Venereology: Chinese Medical Association. Clinical guidelines of psoriasis 2008. Chin J Dermatol (Chin). 2009:42:213-4

32. Altman DG, Dore CJ. Randomisation and baseline comparisons in clinical trials. Lancet. 1990;335:149-53.

33. Standardization Administration of China. Nomenclature and location of auricular points: national standard of People's Republic of China. Beijing: China Standard Publishing House; 2008.

34. Peng L, Mu K, Liu A, et al. Transauricular vagus nerve stimulation at auricular acupoints Kindey (CO10), Yidan (CO11), Liver (CO12) and Shenmen (TF4) can induce auditory and limbic cortices activation measured by fMRI. Hear Res. 2018;359:1-12.

35. Cheung MC, Chan AS, Yip J. Microcurrent stimulation at shenmen acupoint facilitates EEG associated with sleepiness and positive mood: a randomized controlled electrophysiological study. Evid Based Complement Alternat Med. 2015;2015:182837.

36. Zhang SH, Liu YF, Liu B, et al. Prevention of procedural pain in dressing changes of burn wounds by auricular injection of small-dose fentanyl. Zhongguo Zhen Jiu. 2014;34(10):972-4.

37. Zhang CS, Yang AW, Zhang AL, Fu WB, Thien FU, Lewith G, et al. Earacupressure for allergic rhinitis: a systematic review. Clin Otolaryngol. 2010;35:6-12.

38. Yeh ML, Tsou MY, Lee BY, Chen HH, Chung YC. Effects of auricular acupressure on pain reduction in patient-controlled analgesia after lumbar spine surgery. Acta Anaesthesiol Taiwanica. 2010;48(2):80-6

39. National Clinical Guideline Centre (UK). Psoriasis: assessment and management of psoriasis. London: Royal College of Physicians (UK); 2012.

40. Carlin CS, Feldman SR, Krueger JG, Menter A, Krueger GG. A 50\% reduction in the Psoriasis Area and Severity Index (PASI 50) is a clinically significant endpoint in the assessment of psoriasis. J Am Acad Dermatol. 2004;50:859-66.

41. Gordon KB, Feldman SR, Koo JY, Menter A, Rolstad T, Krueger G. Definitions of measures of effect duration for psoriasis treatments. Arch Dermatol. 2005; 141(1):82-4

42. Menter A, Abramovits W, Colón LE, Johnson LA, Gottschalk RW. Comparing clobetasol propionate $0.05 \%$ spray to calcipotriene $0.005 \%$ betamethasone dipropionate $0.064 \%$ ointment for the treatment of moderate to severe plaque psoriasis. J Drugs Dermatol. 2009;8(1):52-7.

43. Sedgwick P. What is intention to treat analysis? BMJ. 2013;346:f3662

44. AbuHilal M, Ho N. Successful treatment of severe psoriasis in an adolescent with ustekinumab. Pediatr Dermatol. 2015:32(3):377-80.

45. Carey W, Glazer S, Gottlieb AB, Lebwohl M, Leonardi C, Menter A, et al. Relapse, rebound, and psoriasis adverse events: an advisory group report. J Am Acad Dermatol. 2006;54(Suppl 1):S171-81

46. Singh SK, Rai T. Relapse in psoriasis with two different tapering regimens of methotrexate: a randomized open-label controlled study. Indian J Dermatol Venereol Leprol. 2015;81(2):144-7.

47. Kaur I, Handa S, Kumar B. Natural history of psoriasis: a study from the Indian subcontinent. J Dermatol. 1997;24:230-4.

48. Pathirana D, Ormerod AD, Saiag P, Smith C, Spuls PI, Nast A, et al. European S3-guidelines on the systemic treatment of psoriasis vulgaris. J Eur Acad Dermatol Venereol. 2009:23:1-70.
49. Heydendael VM, Spuls PI, Opmeer BC, de Borgie CA, Reitsma JB, Goldschmidt WF, et al. Methotrexate versus cyclosporine in moderate to severe chronic plaque psoriasis. N Engl J Med. 2003;349:658-65.

50. Zhang CS, Yu JJ, Parker S, et al. Oral Chinese herbal medicine combined with pharmacotherapy for psoriasis vulgaris: a systematic review. Int J Dermatol. 2014;53:1305-18.

51. Deng S, May BH, Zhang AL, et al. Phytotherapy in the management of psoriasis: a review of the efficacy and safety of oral interventions and the pharmacological actions of the main plants. Arch Dermatol Res. 2014;306:211-29.

52. Talbott W, Duffy N. Complementary and alternative medicine for psoriasis: what the dermatologist needs to know. Am J Clin Dermatol. 2015;16(3):147-65.

53. You E, Kim D, Harris R, et al. Effects of auricular acupressure on pain management: a systematic review. Pain Manag Nurs. 2019;20(1):17-24.

54. Yang LH, Duan PB, Hou QM, et al. Efficacy of auricular acupressure for chronic low back pain: a systematic review and meta-analysis of randomized controlled trials. Evid Based Complement Alternat Med. 2017; 2017:6383649.

55. Yeung WF, Chung KF, Poon MM, et al. Acupressure, reflexology, and auricular acupressure for insomnia: a systematic review of randomized controlled trials. Sleep Med. 2012:13(8):971-84.

56. Chen ML, Tan JY, Suen LK. Auricular therapy for lactation: a systematic review. Complement Ther Clin Pract. 2017:29:169-84.

57. Yeh ML, Ko SH, Wang MH, et al. Acupuncture-related techniques for psoriasis: a systematic review with pairwise and network meta-analyses of randomized controlled trials. J Altern Complement Med. 2017;23(12):930-40.

58. Xiang $Y$, Wu X, Lu C, Wang K. An overview of acupuncture for psoriasis vulgaris, 2009-2014. J Dermatolog Treat. 2017;28(3):221-8.

59. Qing H, Tian YS, Fan JM, Tang BY, Niu HQ, Lu JY. Meridian three-combined therapy for treatment of ordinary psoriasis: a multi-center randomized controlled study. Zhongguo Zhen Jiu. 2009;29(3):181-4.

60. Lu CJ, Xiang Y, Xie XL, Xuan ML, He ZH. A randomized controlled singleblind clinical trial on 84 outpatients with psoriasis vulgaris by auricular therapy combined with optimized Yinxieling formula. Chin J Integr Med. 2012;18(3):186-91

\section{Publisher's Note}

Springer Nature remains neutral with regard to jurisdictional claims in published maps and institutional affiliations.

Ready to submit your research? Choose BMC and benefit from:

- fast, convenient online submission

- thorough peer review by experienced researchers in your field

- rapid publication on acceptance

- support for research data, including large and complex data types

- gold Open Access which fosters wider collaboration and increased citations

- maximum visibility for your research: over $100 \mathrm{M}$ website views per year

At $\mathrm{BMC}$, research is always in progress.

Learn more biomedcentral.com/submissions 